\title{
Stereotactic Radiosurgery for Treatment of Drug-Resistant Epilepsy: State of the Art and Emerging Applications
}

\author{
Pasquale Striano ${ }^{1}$, Marianna Pezzella, Mariya Malova, Alessandro Consales, Pantaleo \\ Romanelli $^{3}$ \\ 1. University of Genova, University of Genova/Gaslini Chidren Hospital 2. 3. Cyberknife Center, Cdi, \\ Milan; European Synchrotron Radiation Facility (esrf), Grenoble, France, AB Medica, Milan;cyberknife \\ Center, Cdi, Milan; European Synchrotron Radiation Facility (esrf), Grenoble, France
}

$\square$ Corresponding author: Pasquale Striano, strianop@gmail.com

Disclosures can be found in Additional Information at the end of the article

\section{Abstract}

Radiosurgery is an emerging and interesting treatment option for several types of drugresistant epilepsies. Particularly, CyberKnife radiosurgery may represent a therapeutic approach for mesial temporal lobe epilepsies, as well as epilepsies due to hypothalamic hamartomas and arteriovenous malformations. There are many reports in the literature, but precise patient selection, dosimetry, target volume, and expected short- and long-term outcomes are still under investigation. We provide an overview of current and emerging radiosurgical technology, to discuss the biological mechanisms and the clinical applications, and general results of radiosurgery for the treatment of drug-resistant epilepsies. Despite encouraging preliminary results, definitive evidence to support the role of radiosurgery in the treatment of drug-resistant epilepsy is missing and more prospective trials are needed to identify predictors for treatment success in the different types of medically intractable epilepsy.

Categories: Neurology, Pediatric Surgery, Neurosurgery

Keywords: epilepsy, radiosurgery, gamma-knife, cyberknife, drug-resistance

\section{Introduction And Background}

Radiosurgery is an attractive approach for the treatment of medically intractable epileptogenic foci. Invasive mapping using depth or subdural electrodes is viewed by many experts in the field as an essential step to localize precisely the focus of seizure onset, especially if a neocortical localization is involved. This approach requires in most cases repeated craniotomies and can be associated with serious complications, such as brain damage, haemorrhage, and infections.

Received 11/09/2013 Review began 11/10/2013 Published 11/25/2013

\section{(c) Copyright 2013}

Striano et al. This is an open access article distributed under the terms of the Creative Commons Attribution License CC-BY 3.0., which permits unrestricted use, distribution, and reproduction in any medium, provided the original author and source are credited.
Cases of failed seizure focus localization or overlap of the focus with eloquent cortex are not uncommon, leading to an aborted resection despite the invasiveness of the entire process. There is an increasing interest toward non-invasive seizure focus mapping using functional neuroimaging techniques, such as single-photon emission computed tomography (SPECT), positron emission tomography (PET), functional magnetic resonance imaging (fMRI), and magnetoencephalography (MEG). The ability to non-invasively map a focus and then proceed to a radiosurgical treatment is a novel and rather attractive avenue for the treatment of drugresistant epilepsy, replacing an invasive and risky approach with a thoroughly non-invasive treatment. Drug-resistant seizures associated with mesial temporal sclerosis (MTS) do not commonly require invasive monitoring, providing a logical target to a non-invasive radiosurgical treatment. Radiosurgery is also an attractive treatment option when the seizure focus is located in eloquent cortex or in surgically challenging brain regions (such as the 
hypothalamus).

Current radiosurgical experience in the treatment of focal epilepsy is mainly based on the Gamma Knife treatment of lesional epilepsy related to arteriovenous malformations (AVM), cavernomas, and tumors. Growing radiosurgical experience is available for the treatment of epilepsy associated with mesial temporal sclerosis and hypothalamic hamartoma [1-2]. Most of the literature is based on retrospective uncontrolled studies. Precise patient selection, dosimetry, target volume, and expected short- and long-term outcomes are still under investigation. Despite the limits of current understanding, the results obtained so far are quite promising. The aim of this paper is to provide an overview of current and emerging radiosurgical technology, to review the biological basis of the radiosurgical treatments for epilepsy, and to discuss the clinical applications and general results of radiosurgery for the treatment of drug-resistant epilepsy.

\section{Review}

\section{Stereotactic radiosurgery: an overview}

Radiosurgery involves the application of focused radiation to a brain target visible on dedicated neuroimaging. Initially conceptualized by Leksell for use in functional neurosurgery, radiosurgical treatment is now considered for a greatly expanding number of neoplastic and vascular indications [3-4]. In fact, radiosurgery allows the neurosurgeon to deliver a precise and accurate amount of radiation to a selected lesional volume without affecting nearby normal parenchyma, and with minimal risk to neurological function [5-7]. Radiosurgery has been historically delivered using the Gamma Knife, a device originally developed by Lars Leksell in Sweden and then widely popularized by the work of L. Dade Lunsford and his school in Pittsburgh. Gamma Knife requires head immobilization through a stereotactic frame in order to focus multiple beams originating from cobalt sources to the selected target. Linac radiosurgery has been developed almost in parallel to Gamma Knife radiosurgery and has not, for a while, achieved the same popularity. Linac radiosurgery is a gantry-based approach where the radiation source rotates around the target. Again, head fixation through a stereotactic frame is essential to provide precision and accuracy to the treatment.

The CyberKnife is a frameless LINAC radiosurgery system characterized by a non-isocentric beam delivery pattern that uses a maneuverable robotic arm which can move the lightweight LINAC around the patient. CyberKnife radiosurgery provides a frameless image-guided treatment of proven accuracy for intra- and extracranial targets [8]. CyberKnife image-guided beam delivery is based on amorphous silicon detector technology providing real-time imaging of the patient's anatomy, which is then overlapped on digitally reconstructed scans from a preoperative CT scan. The position of the target in the space is thus obtained, driving the robotic delivery with submillimetric accuracy. It is therefore possible to deliver conformal highdose radiation with steep gradients, while adding a further protection to nearby radiosensitive tissues through the ability to fractionate the radiation delivery. Hypofractionation is a novel way to deliver radiosurgical high-dose treatments to cranial and extracranial targets, not easily practised when using frame-based approaches but greatly facilitated by the introduction in the clinical field of the CyberKnife [8]. Hypofractionated treatments have not been applied to epilepsy so far, but this additional possibility offered by the CyberKnife can substantially reduce the risks related to the treatment, especially when volumes approaching or larger than 7 cc are treated. CyberKnife radiosurgery provides a thoroughly non-invasive treatment and is especially suited for children due to the absence of a stereotactic frame and to the reduced brain exposure to radiation due to the ability to exploit beam trajectories going through the splanconocranium and skull base, which cannot be used when a head frame is placed, and provides a thoroughly non-invasive treatment. 


\section{Biological effect of irradiation on seizures}

Destruction of the epileptic focus and its pathway of spread by necrotizing radiosurgical doses or, alternatively, suppression of the epileptic activity by a neuromodulatory effect at nonnecrotizing doses have been postulated as the basis of the anti-epileptic effect of focal irradiation [9-10]. Seizures induced by AVMs are often ameliorated after radiosurgical treatment; however, the antiepileptic effect is not directly related to AVM nidus occlusion following radiosurgery [11-13] but appears rather related to irradiation with lower, nonnecrotizing doses of the epileptogenic cortex surrounding the nidus. Experimental models of hippocampal radiosurgery with Gamma Knife have been developed to improve radiobiological understanding of their effects of epileptogenic cortex irradiation in rats. The experimental evidence suggests that, in rats, it is possible to achieve seizure control with subnecrotic doses of radiation. In studies in which an initial dose of $20 \mathrm{~Gy}$ was used, seizure abolition was reported in over $50 \%$ of the treated animals, with increasing seizure control associated with higher doses [14-19]. Overall, these studies suggest that a dose between 20 and 60 Gy given to rats provide seizure amelioration without frank histological necrosis. This therapeutic window is likely to be much narrower in humans, where doses superior to $25 \mathrm{~Gy}$ delivered to the mesial temporal structures are likely to induce necrosis and massive edema. In general, research and clinical efforts made so far to control seizures with stereotactic irradiation have focused on the ability to distribute a relatively homogeneous dose of radiation to a selected cortical volume characterized as the seizure focus. A novel experimental approach under study provides the ability to deliver very high doses (up to several hundred Gy's) through an array of microscopic synchrotron-generated beams. Microbeam irradiation cuts through the tissue in a quasisurgical way parcelizing and disconnecting the focus without injury to the tissue included within the beams. Cortical function is thus preserved, while the critical mass required to the seizure focus to generate epileptic activity is lost and its connections to nearby tissue are severed. Synchrotron-generated microbeam transections over sensorimotor cortex in rats with status epilepticus induced by focal injection of kainic acid induced seizure control without neurological damage [20].

\section{Clinical applications of radiosurgery for epilepsy}

In 1994, Barcia, et al. published the first dedicated report showing the effect of radiosurgery on seizure control. The study reported 11 patients who had epileptic foci treated with doses ranging from 10 to $20 \mathrm{~Gy}$ [21]. In the late 1990s, two papers reported seizure reduction after Gamma Knife radiosurgery of brain tumors [22-23]. The studies included 23 patients with temporal lobe lesions and 24 patients with extratemporal lesions and yielded encouraging results in terms of improved seizure control and freedom. Despite the limitations associated with these studies, positive results in terms of good seizure outcomes in association with very low complication rates aroused interest in the possible role of radiosurgery for the treatment of lesional epilepsy, especially for lesions located in challenging or inoperable brain regions.

\section{Hypothalamic hamartomas}

Hypothalamic hamartomas $(\mathrm{HH})$ are rare, deeply located lesions surrounded by a delicate vascular and neuronal network. They are associated with precocious puberty, cognitive delay, and gelastic epilepsy [24-25]. Patients may experience gelastic or, more rarely, dacrystic seizures as well as dyscognitive seizures, with or without secondary generalization. Disease evolution is generally unfavourable because of the occurrence of several types of seizures, worsening of electroencephalographic (EEG) features, and mental impairment. Microsurgical resection of $\mathrm{HHs}$ has been reported to improve the control of gelastic seizures, but the difficulties in reaching the deep lesions have resulted in a high risk of complications, such as oculomotor nerve palsy, hemiparesis, visual field deficit, and metabolic disturbances related to hypothalamic damage [24-28]. Therefore, alternative treatments, including radiofrequency 
ablation or implantation of radioactive seeds, have been developed.

As a result of being able to deliver conformal high-dose radiation with steep gradients without exceeding tolerance of nearby critical structures, radiosurgery seems to offer an excellent chance in the treatment of patients with HHs in terms of seizure freedom without hypothalamic and cranial nerve damage. Regis, et al. treated 10 patients affected by medically refractory epilepsy associated with HH by means of Gamma Knife radiosurgery [2]. All patients had improved seizure control after radiosurgery. The study showed a correlation between efficacy and dose. The marginal dose was more than $17 \mathrm{~Gy}$ for all patients in the successful group and less than $13 \mathrm{~Gy}$ for all patients in the improved group. The observation that seizure control is strictly dependent on the marginal dose has been confirmed by Unger, et al. who used low marginal doses (12-14 Gy) that resulted in decreased seizure frequency and intensity but no seizure freedom [29]. Dunoyer and coworkers described two children aged four and five years with seizures associated with HHs, treated with Gamma Knife radiosurgery and receiving $11 \mathrm{~Gy}$ to the $85 \%$ and $14 \mathrm{~Gy}$ to the $45 \%$ isodose. The patients showed, respectively, a $90 \%$ reduction in seizure frequency and complete seizure freedom [30]. Recently, De Salles, et al. studied the efficacy of radiosurgery for gelastic seizures. Three patients were treated with 15-18 Gy doses. Two patients became seizure-free and the third patient experienced a substantial reduction in seizure frequency [31]. The results of these studies suggest that radiosurgery is a safe and effective option in the treatment of seizures associated with HHs. This treatment option has a much more benign neurological complication pattern compared to transcranial surgery and therefore should be recommended as first line treatment. Using Gamma Knife radiosurgery, 17 Gy or higher marginal doses are required to achieve seizure control. CyberKnife radiosurgery, providing a frameless approach of great additional value in the treatment of children, has been recently described as a novel treatment option for $\mathrm{HH}$ [32].

\section{Mesial temporal lobe epilepsy}

Mesial temporal lobe epilepsy (MTLE) associated with hippocampal sclerosis (HS) is perhaps the most well-defined epilepsy syndrome that is responsive to surgical treatment, with surgical cure expected in 65-99\% of patients [33-40]. Recently, radiosurgery has been explored as an alternative to open resective surgery for MTS-associated MTLE. In 1995, Regis, et al. first reported selective amygdala-hippocampal radiosurgery for MTLE [41]. This experience has been recently expanded with the results of a prospective multicentric trial. Patients were selected for the Gamma Knife procedure according to the same criteria used for microsurgical amygdalohippocampectomy, including the presence of hippocampal sclerosis and the absence of space-occupying lesions. The results demonstrated the same seizure reduction efficacy rates (65\%) for radiosurgery and for conventional surgery at two years of follow-up [1]. Using a marginal dose of $24 \mathrm{~Gy}$, the study again demonstrated that radiosurgery may be used as an alternative to resective surgery to treat MTLE associated with MTS and to improve quality of life with favourable rates of morbidity and mortality. In the US, a multicentric pilot trial is under way to evaluate the safety of the Gamma Knife procedure in patients with MTLE and to determine the appropriate radiation dose in a larger phase III trial. After two years' follow-up, $85 \%$ of patients treated with $24 \mathrm{~Gy}$ (to the $50 \%$ isodose line) to the medial temporal lobe were seizure-free with minimal morbidity (unpublished data). Quality-of-life assessments at two to three years showed improvements that parallel freedom from seizure. This trial was not designed to define the true efficacy of radiosurgery in MTLE versus open surgery. A phase III trial for patients with clinically and radiographically-defined MTS has been planned. All studies on the efficacy of radiosurgery showed that the beneficial effects do not appear immediately. In fact, most patients achieved seizure reduction at nine to 12 months and complete cessation of seizures occurred between 18 and 24 months after radiosurgical treatment. Recent studies in small numbers of patients suggested that a lower marginal dose of $20 \mathrm{~Gy}$ may be less effective in reducing seizures. Cmelak, et al. reported unsuccessful seizure reduction with radiosurgery using a 15 Gy dose [42]. Kawai, et al. also reported two cases of unsuccessful treatment with an 
18 Gy dose [43]. Finally, Srikijvilaikul, et al. reported on a series in which Gamma Knife radiosurgical treatment with a $20 \mathrm{~Gy}$ marginal dose failed in seizure control [44]. Clearly, larger numbers of patients followed over a longer period of time would be required to determine the true percentage of seizure-free outcomes with this dose. Successful radiosurgical treatment has also been shown to be target-related. Regis, et al. radiosurgically targeted the mesial temporal lobe structures (including amygdala, anterior hippocampus, and nearby cortex) in a series of patients, whereas Kavay, et al. confined their treatment to the amygdala or hippocampus [1, 4346]. The results of each series show that targeting the entire mesial temporal lobe may be a more effective method of reducing seizures. Although target definition may be variable among different neurosurgeons, radiosurgery for MTS-associated MTLE represents an attractive option because of its low morbidity and mortality.

\section{Epilepsy related to arteriovascular malformations}

The analysis of seizure outcome is extremely complex in patients with epilepsy related to arteriovascular malformations (AVM) because of the inadequate and retrospective design of most studies. We review here some major relevant reports, even though it is difficult to draw definitive conclusions regarding efficacy of radiosurgery treatment. Studies carried out over the past 25 years on seizure outcome after radiosurgery with Gamma Knife, LINAC and photon beams are most notable for the wide range of reported seizure outcomes, and show improvement rates varying from $85 \%$ to $20 \%$, or no improvement at all [12, 47-48]. In some cases, radiosurgery induced a deterioration of seizure control, while recent papers showed good seizure outcomes [11-13, 49-53]. The reduction of seizure frequency and intensity in patients with AVM undergoing surgery seems to be independent of nidus obliteration. Steiner, et al. reported that 41 of 59 patients with seizures became seizure-free or showed significant improvement after radiosurgery. AVM obliteration was not needed to achieve seizure freedom [11]. In a series of 100 patients with AVMs treated with LINAC radiosurgery, Eisenshenk, et al. found that 59 patients were seizure-free and 19 had a substantial reduction in frequency [13]. Again, there was no association between thrombosis of the nidus and seizure outcome, which confirmed the observations made by Stainer. A report by Kurita, et al. also seems to confirm this speculation [50]. Kida, et al studied 462 patients with cerebral AVMs treated with Gamma Knife radiosurgery with a marginal dose of $19.8 \mathrm{~Gy}$. The overall results indicate that seizures improved in $85 \%$ of cases, remained unchanged in $12 \%$, and worsened in $3 \%$ [12]. Radiosurgery was effective in seizure control even before complete occlusion of the nidus. Another retrospective study by Hadyipanayis, et al. reporting the outcome of Gamma Knife surgery for precentral gyrus AVM showed that good seizure outcome and low morbidity are possible when treating high-risk lesions. The median dose to the margin was $20 \mathrm{~Gy}$. After a follow-up of 4.5 years, $63 \%$ of patients were seizure-free, while the remaining $37 \%$ continued to have seizures at a frequency not higher than that observed in radiosurgery [52]. Schauble, et al. identified 70 patients with seizures associated with AVM who had been treated with Gamma Knife radiosurgery [53]. Average prescribed dose was $18 \mathrm{~Gy}$. Sixty-five patients were followed up for one year and 51 patients for three years. At one year and at three years, seizure rates were $45 \%$ and $51 \%$, respectively. One patient died due to radiation-induced edema. Overall seizure improvement is quite common in patients with AVM treated with radiosurgery and rivals the results yielded by microsurgical resection. The limited morbidity and good outcomes associated with the radiosurgical treatment of epileptogenic AVM located close to or within the eloquent cortex makes radiosurgical treatment a valid alternative option.

\section{Cavernous malformation}

A cavernous malformation $(\mathrm{CM})$ is a congenital vascular abnormality that can cause haemorrhage or neurological deficit but more commonly manifests as recurring seizures [5455]. Although open microsurgical treatment of CM remains the standard efficacious therapy, a recent study by Regis, et al. suggested a role for radiosurgery in the treatment of seizures 
associated with CM near the "highly functional cortex", a location that may preclude open resection [54]. Using a mean dose of $19 \mathrm{~Gy}, 53 \%$ of 49 patients with refractory seizures became seizure-free and $20 \%$ of treated ones improved at two years [54]. These results demonstrate that epilepsy associated with CM located near the eloquent cortex may be treated with radiosurgery to reduce seizure frequency. However, deeply-seated CM carries a high risk of clinically evident bleeding associated with a poor neurologic outcome [56], and therefore the role of radiosurgery in these cases is still unclear. Without clear evidence of the effect of radiosurgery on the risk of bleeding, microsurgical resection remains the standard therapy for CM.

\section{Risk profile of stereotactic radiosurgery}

Radiosurgery is, in most cases, a well-tolerated procedure with minimal morbidity. Case selection, imaging and dose protocols, training, and experience of the team involved in the treatment are essential to maintain this low risk profile. Short to intermediate-term risks of radiosurgery are modest. The long-term risks of vascular complications and secondary neoplasms are low but difficult to quantify. One analysis estimated the risk of secondary neoplasms at zero to three per 200,000 patients; however, other authors suggested that the prevalence might be somewhat higher [57-59]. Since radiation-induced neoplasms require decades to develop, long-term investigations are required. The long-term risks and outcomes specifically for epilepsy are not known yet.

\section{Conclusions}

Radiosurgery is an emerging treatment option for several types of medically-intractable epilepsy, including MTLE, as well as epilepsy due to HH and AVM. CyberKnife radiosurgery may represent a therapeutic leap forward thanks to its ability to provide a frameless but still highly conformal and accurate treatment with the additional protection given by hypofractionation in selected cases. Despite encouraging preliminary results, definitive evidence to support the role of radiosurgery in the treatment of drug-resistant epilepsy is missing. Prospective trials with larger numbers of patients are required in order to establish radiosurgery as a standard therapy in the different types of epilepsy.

\section{Additional Information}

\section{Disclosures}

Conflicts of interest: In compliance with the ICMJE uniform disclosure form, all authors declare the following: Payment/services info: All authors have declared that no financial support was received from any organization for the submitted work. Financial relationships: All authors have declared that they have no financial relationships at present or within the previous three years with any organizations that might have an interest in the submitted work. Other relationships: All authors have declared that there are no other relationships or activities that could appear to have influenced the submitted work.

\section{References}

1. Regis J, Rey M, Bartolomei F, et al.: Gamma Knife surgery in mesial temporal lobe epilepsy: A prospective multicenter study. Epilepsia. 2004, 45:504-15.

2. Regis J, Bartolomei F, de Toffol B, et al.: Gamma knife surgery for epilepsy related to hypothalamic hamartomas. Neurosurgery. 2000, 47:1943-52.

3. Kitchen N: Experimental and clinical studies on the putative therapeutic efficacy of cerebral irradiation (radiotherapy) in epilepsy. Epilepsy Res. 1995, 20:1-10.

4. Sun B, DeSalles AA, Medin PM, et al.: Reduction of hippocampal kindled seizure activity in rats by stereotactic radiosurgery. Exp Neurol. 1998, 154:691-695.

5. Nguyen DK, Spencer SS: Recent advances in the treatment of epilepsy. Arch Neurol. 2003, 
60:929-935.

6. Kondziolka D, Lunsford LD, Witt TC, Flickinger JC: The future of radiosurgery: Radiobiology, technology, and applications. Surg Neurol. 2000, 54:406-414.

7. Dillon WP, Barbaro N: Noninvasive surgery for epilepsy: The era of image guidance . AM J Neuroradiol. 1999, 20:185.

8. Romanelli P, Adler JR: Technology Insight: Image-guided robotic radiosurgery-A new approach for non-invasive ablation of spinal lesions. Nat Clin Pract Oncol. 2008, 5:405-14.

9. Regis J, Kerkerian-Legoff L, Rey M, et al.: First biochemical evidence of differential functional effects following gamma knife surgery. Stereotact Funct Neurosurg. 1996, 66:29-38.

10. Romanelli P, Striano P, Barbarisi M, Coppola G, Anschel DJ: Non-resective surgery and radiosurgery for treatment of drug-resistant epilepsy. Epilepsy Res. 2012, 99:193-201.

11. Steiner L, Lindquist C, Adler JR, Torner JC, Alves W, Steiner M: Clinical outcome of radiosurgery for cerebral arteriovenous malformations. J Neurosurg. 1992, 77:1-8.

12. Kida Y, Kobayashi T, Tanaka T, Mori Y, Hasegawa T, Kondoh T: Seizure control after radiosurgery on cerebral arteriovenous malformations. J Clin Neurosci. 2000, 7:6-9.

13. Eisenschenk S, Gilmore RL, Friedman WA, Henchey RA: The effect of LINAC stereotactic radiosurgery on epilepsy associated with arteriovenous malformations. Stereotact Funct Neurosurg,. 1998, 71:51-61.

14. Maesawa S, Kondziolka D, Dixon C, Balzer J, Fellows W, Lunsford LD: Subnecrotic stereotactic radiosurgery controlling epilepsy produced by kainic acid injection in rats. J Neurosurg. 2000, 93:1033.

15. Mori Y, Kondziolka D, Balzer J, et al.: Effects of stereotactic radiosurgery on an animal model of hippocampal epilepsy. Neurosurgery. 2000, 46:57-68.

16. Chen ZF, Kamiryo T, Henson SL et al.: Anticonvulsant effects of gamma surgery in a model of chronic spontaneous limbic epilepsy in rats. J Neurosurg. 2001, 94:270-80.

17. Liscak R, Vladyka V, Novotny J, et al.: Leksell Gamma Knife lesioning of the rat hippocampus: The relationship between radiation dose and functional and structural damage. J Neurosurg. 2002, 97:666-73.

18. Brisman JL, Cole AJ, Cosgrove GR, et al.: Radiosurgery of the rat hippocampus: Magnetic resonance imaging, neurophysiological, histological, and behavioral studies. Neurosurgery. 2003, 53:951-62.

19. Jenrow KA, Ratkewicz AE, Lemke NW, et al.: Effects of kindling and irradiation on neuronal density in the rat dentate gyrus. Neurosci Lett. 2004, 371:45-50.

20. Romanelli P, Fardone E, Battaglia G, et al.: Synchrotron-generated microbeam sensorimotor cortex transections induce seizure control without disruption of neurological functions. PLoS One. 2013, 8:e53549. 10.1371/journal.pone.0053549

21. Barcia JA, Barcia-Salorio JL, Lopez-Gomez L, Hernandez G: Stereotactic radiosurgery may be effective in the treatment of idiopathic epilepsy: Report on the methods and results in a series of eleven cases. Stereotact Funct Neurosurg. 1994, 63:271-79.

22. Whang CJ, Kwon Y: Long-term follow up of stereotactic Gamma Knife radiosurgery in epilepsy. Stereotact Funct Neurosurg, . 1996, 66:349-56.

23. Schrottner O, Eder HG, Unger F, Feichtinger K, Pendl G: Radiosurgery in lesional epilepsy: Brain tumors. Sterotact Funct Neurosurg. 1998, 70:50-56.

24. Regis J, Bartolomei F, de Toffol B, Genton P, Kobayashi T, Mori Y, Takakura K, Hori T, Inoue H, Schrottner O, Pendl G, Wolf A, Arita K, Chauvel P: Gamma Knife surgery for epilepsy related to hypothalamic hamartomas. Neurosurgery. 2000, 47:1343-1351.

25. Striano S, Santulli L, Ianniciello M, Ferretti M, Romanelli P, Striano P: The gelastic seizureshypothalamic hamartoma syndrome: Facts, hypotheses, and perspectives. Epilepsy Behav. 2012, 24:7-13.

26. Striano S, Striano P, Coppola A, Romanelli P: The syndrome gelastic seizures-hypothalamic hamartoma: Severe, potentially reversible encephalopathy. Epilepsia,. 2009, 50:62-5.

27. Fohlen M, Lellouch A, Delalande O: Hypothalamic hamartoma with refractory epilepsy: surgical procedures and results in 18 patients. Epileptic Disord. 2003, 5:267-273.

28. Delalande O, Fohlen M: Disconnecting surgical treatment of hypothalamic hamartoma in children and adults with refractory epilepsy and proposal of a new classification. Neurol Med Chir (Tokyo),. 2003, 43:61-68.

29. Unger F, Schrottner O, Feichtinger M, Bone G, Haselsberger K, Sutter B: Stereotactic radiosurgery for hypothalamic hamartomas. Acta Neurochirurgica. 2002, 84:57-63. 
30. Dunoyer C, Ragheb J, Resnick T, et al.: The use of stereotactic radiosurgery to treat intractable childhood partial epilepsy. Epilepsia. 2002, 43:292-300.

31. Selch MT, Gorgulho A, Mattozo C, Solberg TD, Cabatan-Awang C, De Salles AA: Linear accelerator stereotactic radiosurgery for the treatment of gelastic seizures due to hypothalamic hamartoma. Minim Invasive Neurosurg. 2005, 48:310-14.

32. Romanelli P, Muacevic A, Striano S: Radiosurgery for hypothalamic hamartomas. Neurosurgical Focus. 2008, 24:E9.

33. Bien CG, Kurthen M, Baron K, Lux S, Helmstaedter C, Schramm J, Elger CE: Long-term seizure outcome and antiepileptic drug treatment in surgically treated temporal lobe epilepsy patients: a controlled study. Epilepsia. 2001, 42:1416-21.

34. Engel Jr J: Finally, a randomized, controlled trial of epilepsy surgery. N Engl J Med. 2001, 345:365-67.

35. Spencer SS, Berg AT, Vickrey BG, Sperling MR, Bazil CW, Shinnar S, Langfitt JT, Walczak TS, Pacia SV, Ebrahimi N, Frobish D: Initial outcomes in the multicenter study of epilepsy surgery. Neurology. 2003, 61:1680-85.

36. Wiebe S, Blume WT, Girvin JP, Eliasziw M: A randomized, controlled trial of surgery for temporal-lobe epilepsy. N Engl J Me. 2001, 345:311-18.

37. Engel Jr J: Update on surgical treatment of the epilepsies, Summary of the second International Palm Desert Conference on the surgical treatment of the epilepsies (1992). Neurology. 1993, 43:1612-17.

38. Cascino GD: Clinical correlations with hippocampal atrophy. Magn Reson Imaging. 1995, 13:1133-36.

39. Cascino GD: Structural neuroimaging in partial epilepsy. Magnetic resonance imaging. Neurosurg Clin N Am. 1995, 6:455-64.

40. Garcia PA, Laxer KD, Barbaro NM, Dillon WP: Prognostic value of qualitative magnetic resonance imaging hippocampal abnormalities in patients undergoing temporal lobectomy for medically refractory seizures. Epilepsia. 1994, 35:520-24.

41. Regis J, Bartolomei F: Comment on: Failure of Gamma Knife radiosurgery for mesial temporal lobe epilepsy: report of five cases. Neurosurgery. 2004, 54:1404.

42. Cmelak AJ, Abou-Khalil B, Konrad PE, Duggan D, Maciunas RJ: Low-dose stereotactic radiosurgery is inadequate for medically intractable mesial temporal lobe epilepsy: A case report. Seizure. 2001, 10:442-46.

43. Kawai K, Suzuki I, Kurita H, Shin M, Arai N, Kirino T: Failure of low-dose radiosurgery to control temporal lobe epilepsy. Neurosurg. 2001, 95:883-87.

44. Srikijvilaikul T, Najm I, Foldvary-Schaefer N, Lineweaver T, Suh JH, Bingaman WE: Failure of Gamma Knife radiosurgery for mesial temporal lobe epilepsy: report of five cases. Neurosurgery. 2004, 54:1395-1402.

45. Regis J, Peragui JC, Rey M, Samson Y, Levrier O, Porcheron D, Regis H, Sedan R: First selective amygdalohippocampal radiosurgery for mesial temporal lobe epilepsy. Stereotact Funct Neurosurg. 1995, 64:193-201.

46. Regis J, Bartolomei F, Rey M, et al.: Gamma Knife surgery for mesial temporal lobe epilepsy . Epilepsia. 1999, 40:1551-56.

47. Coffey RJ, Nichols DA, Shaw EG: Stereotactic radiosurgical treatment of cerebral arteriovenous malformations. Mayo Clin Proc. 1995, 70:214-22.

48. Colombo F, Benedetti A, Pozza F, Marchetti C, Chierego G: Linear accelerator radiosurgery of cerebral arteriovenous malformations. Neurosurgery. 1989, 24:833-39.

49. Friedman WA, Bova FJ, Mendenhall WM: Linear accelerator radiosurgery for arteriovenous malformations: the relationship of size to outcome. J Neurosurg. 1995, 82:180-89.

50. Kurita H, Kawamoto S, Suzuki I, et al.: Control of epilepsy associated with cerebral arteriovenous malformations after radiosurgery. J Neurol Neurosurg Psychiatry. 1998, 65:64855.

51. Hoh BL, Ogilvy CS, Butler WE, Loeffler JS, Putman CM, Chapman PH: Multimodality treatment of nongalenic arteriovenous malformations in pediatric patients. Neurosurgery. 2000, 47:346-58.

52. Hadjipanayis CG, Levy EI, Niranjan A, et al.: Stereotactic radiosurgery for motor cortex region arteriovenous malformations. Neurosurgery. 2001, 48:70-77.

53. Schauble B, Cascino GD, Pollock BE, et al.: Seizure outcomes after stereotactic radiosurgery for cerebral arteriovenous malformations. Neurology. 2004, 63:683-87. 


\section{Cureus}

54. Regis J, Bartolomei F, Kida Y, et al.: Radiosurgery for epilepsy associated with cavernous malformation: Retrospective study in 49 patients. Neurosurgery. 2000, 47:1091-97.

55. Maraire JN, Awad IA: Intracranial cavernous malformations: lesion behavior and management strategies. Neurosurgery. 1995, 37:591-605.

56. Porter PJ, Willinsky RA, Harper W, Wallace MC: Cerebral cavernous malformations: Natural history and prognosis after clinical deterioration with or without hemorrhage. J Neurosur. 1997, 87:190-97.

57. Laws ER, Sheehan JP, Sheehan JM, Jagnathan J, Jane Jr JA, Oskouian R: Stereotactic radiosurgery for pituitary adenomas: A review of the literature. J Neurooncol. 2004, 69:25772 .

58. Ganz JC: Gamma Knife radiosurgery and its possible relationship to malignancy: A review . J Neurosurg. 2002, 97:644-52.

59. Loeffler JS, Niemierko A, Chapman PH: Second tumors after radiosurgery: Tip of the iceberg or a bump in the road. Neurosurgery. 2003, 52:1436-42. 